\title{
Welcoming Address
}

\author{
Petr Harmanec $\dagger$ \\ Astronomical Institute of the Charles University, Faculty of Mathematics and Physics, \\ V Holešovičkách 2, CZ-180 00 Praha 8, Czech Republic \\ email: hec@sirrah.troja.mff.cuni.cz \\ and \\ Astronomical Institute, Academy of Sciences of the Czech Republic, CZ-251 65 Ondřejov, \\ Czech Republic \\ email: hec@sunstel.asu.cas.cz
}

Dear colleagues and friends, ladies and gentlemen,

It is great privilege for me to welcome so many of you to Prague, or Praha as we call our capital in Czech.

We have been witnessing a very rapid development of observational techniques over the past few decades, leading to many exciting discoveries, quite often in the field of extragalactic research. I sometimes sense the tendency to consider the studies of binaries and stars in general as exhausted topics. However, we all know that the better our real and deep understanding of the principal sources of radiative energy in the Universe becomes, the safer will be the ground for studies of all higher hierarchical systems.

I am therefore very pleased to see how great an interest this symposium attracted and I am especially happy to see not only old friends but also many young colleagues in the audience.

As most of you know, there were originally two proposals for this meeting and I find it great that it resulted in this joint effort. Interferometry and other new techniques are gradually removing the distinction between astrometric and spectroscopic binaries, and I am personally very keen to see the progress in this area. In particular, this will give us the chance to derive certain basic properties of stars like the limb- and gravity-darkening of rapidly rotating early-type stars simply via comparison of observed radiative properties of stars of the same mass seen under different aspect angle, from equator-on to pole-on.

Let me also express our appreciation for the help given to us by all members of our Scientific Organizing Committee and our advisers, who were active in all phases of preparation for this meeting. Our apologies at the same time to all of you who were not given the chance to present your favourite topics. Please, consider that we were put into a very uneasy position. We were first promised to have a five-day meeting and while the initially-suggested program was taking shape, the news came that all symposia can go on for only 3.5 days.

Some practical information:

1. It is now obligatory to record the discussions following all invited talks. Our assistants will distribute discussion sheets to everybody who asks a question after a talk and then to the speakers to add their answers. We hope this might naturally regulate your anxiety to discuss! Note also that no discussion will be allowed after the short oral presentations of selected posters, due to time constraints.

2. Thursday evening starting at 6 p.m. Marek Wolf, Miloslav Zejda and I have organized a public outreach: a discussion between professional and amateur astronomers

$\dagger$ Present address: Astronomical Institute of the Charles University, V Holešovičkách 2, CZ-180 00 Praha 8, Czech Republic 
about binary-star observations by amateurs. This discussion will be held at the building of the faculty of Mathematics and Physics of the Charles University, located at the other side of Nuselský bridge. It is within walking distance of here, and we invite all interested people to join us.

Let me end this welcome by saying the following: From the very beginning of my career in astronomy, I always had a very pleasant feeling that the students of binaries all over the world represent a very friendly community joined by their love of binaries and multiple systems. I heartily hope that we all will share the same feeling during our meeting which I now declare open. 